\title{
Recent insights into the structural characterization of herpes simplex virus fusion protein, $\mathrm{gB}$
}

\author{
Lorelai M Vennard ${ }^{1}$, Doina Atanasiu ${ }^{2}$, Wan Ting Saw², Roselyn J Eisenberg ${ }^{3}$, Gary H Cohen ${ }^{2}$ \\ \& Juan Fontana*,1 \\ ${ }^{1}$ Faculty of Biology \& Astbury Center for Structural Molecular Biology, University of Leeds, Leeds LS2 9JT, UK \\ ${ }^{2}$ Department of Microbiology, School of Dental Medicine, University of Pennsylvania, Philadelphia, PA, USA \\ ${ }^{3}$ Department of Pathobiology, School of Veterinary Medicine, University of Pennsylvania, Philadelphia, PA, USA \\ * Author for correspondence: j.fontana@leeds.ac.uk
}

\begin{abstract}
'Using cryo-EM, we imaged vesicles expressing full-length gB bound to monovalent antibody fragments that do not possess an Fc region (Fabs) and to whole antibodies, along with gB containing genetically encoded FP insertions. Since the Fabs, antibodies and FPs were visible by cryo-EM, we used them as landmarks to map the position of $\mathrm{gB}$ domains in its prefusion conformation. According to our experimental data, we proposed that, initially, gB has the fusion loops pointing toward the viral membrane"
\end{abstract}

First draft submitted: 11 October 2017; Accepted for publication: 12 October 2017; Published online: 15 January 2018

The herpesviridae family replicate within a wide range of vertebrate hosts and one invertebrate host. The family includes over 100 viruses, of which eight cause human infections such as herpes simplex virus (HSV). HSV is a model system for the herpesvirus family and has two serotypes, HSV-1 and HSV-2, that globally infect approximately $90 \%$ of the population [1]. HSV inflicts lifelong infections by establishing latency in the host and undergoing periodic reactivations that cause the virus to spread. These infections normally manifest as mucocutaneous infections including keratitis, gingivostomatitis and genital warts. Furthermore, infection with HSV-2 increases the risk of acquiring and transmitting HIV [1]. Specific antivirals limit the impact of HSV but none cure infection. Coupled with the lack of a preventative vaccine, this virus will continue to afflict the population, making it a global health burden of high priority.

HSV has a linear DNA genome of approximately $152 \mathrm{~kb}$ packaged tightly in an icosahedral capsid, which is $15 \mathrm{~nm}$ thick and $125 \mathrm{~nm}$ in diameter. The capsid itself is encased in a matrix of 20 proteins (the tegument), that lies beneath a host-derived lipid envelope surrounded by 10-12 glycoproteins [2]. Therefore, the size and diverse complexity of HSV make structural studies extremely challenging.

As with all enveloped viruses, HSV infection begins with entry, a process that requires fusion of cellular and viral membranes. While the molecular details are still not known, all events are thought to follow the fusion-throughhemifusion pathway [3]. The basic principle posits that the fusion of two lipid membranes is thermodynamically favorable and that the high kinetic barrier is overcome when free energy is released as the fusion protein undergoes a series of conformational changes. These changes bring the membranes in close proximity, inducing membrane curvature, hemifusion (where only the outer leaflets are fused) and finally full fusion [4].

HSV membrane fusion is mediated by four glycoproteins: the primary receptor binding protein gD, a covalently linked heterodimer $\mathrm{gH} / \mathrm{gL}$ and the fusion protein, gB. HSV fusion begins with the interaction of $\mathrm{gD}$ with a cellular receptor. This interaction induces a conformational change in $\mathrm{gD}$, prompting $\mathrm{gH} / \mathrm{gL}$ to activate $\mathrm{gB}$. Successive rearrangements of $\mathrm{gB}$, from its initial metastable prefusion conformation to the more energetically favored postfusion conformation, leads to membrane curvature and disruption of cellular membranes, resulting in viral capsid release into the host cell [5].

Several structures of HSV glycoproteins exist, including unliganded gD, gD in complex with its receptors [6] and a partially activated form of $\mathrm{gH} / \mathrm{gL}$ [7]. However, only the postfusion structure of gB has been solved [8]. This 
is because all purified forms of $\mathrm{gB}$ adopt the postfusion conformation, and attempts to change this have been unsuccessful [9]. This leaves an important gap in the knowledge of the HSV lifecycle.

HSV-1 gB is comprised of 904 residues and acts as a trimer in the postfusion conformation. Side views depict the protein as a three-lobed structure. The truncated postfusion structure identifies five domains, with the two fusion loops residing in domain I. Both domains I and V are at the 'base' of the protein, in close proximity to the viral membrane. Domain II, the central lob, is postulated to mediate interactions with $\mathrm{gH} / \mathrm{gL}$ and is connected to the trimeric coiled-coil domain III. Domain IV, the 'crown', resides at the top, tethered to domain II by domain III [10]. The N-terminus (residues 31-102, putatively domain VI) is not resolved in the crystal structure due to its flexibility. Amino acids 730-904, which are missing in the purified proteins used for crystallographic studies, include the cytoplasmic tail, the transmembrane domain and the membrane-proximal region, all of which are involved in virus fusion and infectivity.

Viral fusion proteins are categorized into three distinct classes: I, II and III. As a class III fusion protein, gB is composed of $\alpha$-helices and $\beta$-sheets, and contains two fusion loops per protomer. Class III fusion proteins are found in herpesviruses, vesicular stomatitis virus (VSV) and baculovirus. The VSV fusion protein, G, is the best characterized class III fusion protein and its postfusion form shares features similar to $\mathrm{gB}[11,12]$. Based on the structures of pre- and postfusion G, Gallagher et al. created an in silico model for prefusion $\mathrm{gB}$ [13,14]. To generate it, they proposed that $\mathrm{gB}$ 's prefusion domain arrangements are similar to $\mathrm{G}$ in its prefusion conformation, and accordingly gB's fusion loops would point toward the viral membrane. Therefore, by analogy to G, during the transition from its pre- to postfusion conformation, the fusion loops would first relocate to the top of $\mathrm{gB}$ to interact with the target membrane. Further conformational changes would position the fusion loops of $\mathrm{gB}$ close to the transmembrane domains, leading to the merging of the cell and virus membranes. This model is supported by an in-depth structural study using fluorescent proteins (FP) to map gB's domains, which suggested that regions allowing insertion of the FPs are exposed [14].

A second model of prefusion $\mathrm{gB}$ was recently proposed by Zeev-Ben-Mordehai et al. [15]. This was generated using cryo-electron microscopy (cryo-EM) to image microvesicles expressing full-length gB. Cryo-EM allows imaging of specimens at atomic or molecular resolution in close-to-native conditions. gB expressed in microvesicles adopted two different conformations: an elongated postfusion form, and a compact form, putatively prefusion $\mathrm{gB}$. They then calculated a $3 \mathrm{D}$ average of the compact form, fitting two postfusion domains of $\mathrm{gB}$ (domains I and II) into the average. Based on VSV G, and like Gallagher et al., they assumed that the domains of gB are similar in the preand postfusion conformations. The resulting model suggests that gB's fusion loops (within domain I) point away from the viral membrane. Therefore, to produce fusion, $\mathrm{gB}$ would extend so that the fusion loops could reach the target membrane, and then conformational changes, similar to the ones proposed by Gallagher et al., would merge the cell and virus membranes.

Recently, we augmented the microvesicle strategy [15] to produce gB in its prefusion form [16]. Using cryo-EM, we imaged vesicles expressing full-length $\mathrm{gB}$ bound to monovalent antibody fragments that do not possess an Fc region (Fabs) and to whole antibodies, along with $\mathrm{gB}$ containing genetically encoded FP insertions. Since the Fabs, antibodies and FPs were visible by cryo-EM, we used them as landmarks to map the position of $\mathrm{gB}$ domains in its prefusion conformation. According to our experimental data, we proposed that, initially, $\mathrm{gB}$ has the fusion loops pointing toward the viral membrane [16], thereby agreeing with the model proposed by Gallagher et al. Additionally, some samples trapped intermediate conformations of $\mathrm{gB}$, providing insights about how the pre- to postfusion transitions could take place. Based on these intermediate conformations, we suggested that the fusion loops of $\mathrm{gB}$, which initially point toward the viral membrane, are relocated to the top of the molecule as a second step in the fusion process, while $\mathrm{gB}$ maintains a compact conformation. This intermediate conformation would therefore be similar to the one proposed by Zeev-Ben-Mordehai et al., reconciling the two models for two conformations of gB. More data will be needed to unequivocally unravel the prefusion structure of $\mathrm{gB}$ and its transition to the postfusion form, thereby elucidating the mechanism of fusion.

In conclusion, although the prefusion structure of HSV gB still poses a challenge to structural biologists, the advances in structural determination techniques and the ability to produce $\mathrm{gB}$ in conformations other than postfusion, are bringing us closer to the answer. This structure will help with rational drug design and vaccine development to tackle HSV infection. 


\section{Financial \& competing interests disclosure}

This work was supported by the University of Leeds (University Academic Fellow scheme) and the National Institutes of Health grant R01-Al-18289 (GH Cohen). The authors have no other relevant affiliations or financial involvement with any organization or entity with a financial interest in or financial conflict with the subject matter or materials discussed in the manuscript apart from those disclosed.

No writing assistance was utilized in the production of this manuscript.

\section{References}

1 WHO. Herpes simplex virus (2017).www.who.int/mediacentre/factsheets/fs400/en/.

2 Grunewald K, Desai P, Winkler DC et al. Three-dimensional structure of herpes simplex virus from cryo-electron tomography. Science 302(5649), 1396-1398 (2003).

3 Chernomordik LV, Kozlov MM. Mechanics of membrane fusion. Nat. Struct. Mol. Biol. 15(7), 675-683 (2008).

4 Harrison SC. Viral membrane fusion. Nat. Struct. Mol. Biol. 15(7), 690-698 (2008).

5 Eisenberg RJ, Atanasiu D, Cairns TM, Gallagher JR, Krummenacher C, Cohen GH. Herpes virus fusion and entry: a story with many characters. Viruses 4(5), 800-832 (2012).

6 Krummenacher C, Carfí A, Eisenberg RJ, Cohen GH. Entry of herpesviruses into cells: the enigma variations. Adv. Exp. Med. Biol. 790, 178-195 (2013).

7 Chowdary TK, Cairns TM, Atanasiu D, Cohen GH, Eisenberg RJ, Heldwein EE. Crystal structure of the conserved herpesvirus fusion regulator complex gH-gL. Nat. Struct. Mol. Biol. 17(7), 882-888 (2010).

8 Stampfer SD, Lou H, Cohen GH, Eisenberg RJ, Heldwein EE. Structural basis of local, pH-dependent conformational changes in glycoprotein B from herpes simplex virus type 1. J. Virol. 84(24), 12924-12933 (2010).

9 Vitu E, Sharma S, Stampfer SD, Heldwein EE. Extensive mutagenesis of the HSV-1 gB ectodomain reveals remarkable stability of its postfusion form. J. Mol. Biol. 425(11), 2056-2071 (2013).

10 Cooper RS, Heldwein EE. Herpesvirus gB: a finely tuned fusion machine. Viruses 7(12), 6552-6569 (2015).

11 Roche S, Albertini AA, Lepault J, Bressanelli S, Gaudin Y. Structures of vesicular stomatitis virus glycoprotein: membrane fusion revisited. Cell Mol. Life Sci. 65(11), 1716-1728 (2008).

12 Roche S, Rey FA, Gaudin Y, Bressanelli S. Structure of the prefusion form of the vesicular stomatitis virus glycoprotein G. Science 315(5813), 843-848 (2007).

13 Atanasiu D, Saw WT, Gallagher JR et al. Dual split protein-based fusion assay reveals that mutations to herpes simplex virus (HSV) glycoprotein gB alter the kinetics of cell-cell fusion induced by HSV entry glycoproteins. J Virol. 87(21), 11332-11345 (2013).

14 Gallagher JR, Atanasiu D, Saw WT et al. Functional fluorescent protein insertions in herpes simplex virus gB report on gB conformation before and after execution of membrane fusion. PLoS Pathog. 10(9), e1004373 (2014).

15 Zeev-Ben-Mordehai T, Vasishtan D, Hernández Durán A et al. Two distinct trimeric conformations of natively membrane-anchored full-length herpes simplex virus 1 glycoprotein B. Proc. Natl Acad. Sci. USA 113(15), 4176-4181 (2016).

16 Fontana J, Atanasiu D, Saw WT et al. The fusion loops of the initial prefusion conformation of herpes simplex virus 1 fusion protein point toward the membrane. MBio 8(4), 1268-1217 (2017). 
